Received: 2016.08.12 Accepted: 2016.10.17 Published: 2017.01.03

\title{
Pituitary Metastasis from Renal Cell Carcinoma: Description of a Case Report
}

Authors' Contribution: Study Design A Data Collection B Statistical Analysis C Data Interpretation D Manuscript Preparation E Literature Search F Funds Collection G
ABCDDEF 1 Chloé Wendel ABCDE 1 Marco Campitiello

$A B C D E 1$ Francesca Plastino

ABCDE 1 Nada Eid

$A B C D E 2$ Laurent Hennequin

$A B C D E 3$ Philippe Quétin

ABCDEFG 1 Raffaele Longo
1 Division of Medical Oncology, Regional Hospital Center (CHR) Metz-Thionville, Ars-Laquenexy, France

2 Division of Radiology, Regional Hospital Center (CHR) Metz-Thionville, Ars-Laquenexy, France

3 Division of Radiotherapy, Regional Hospital Center (CHR) Metz-Thionville, Ars-Laquenexy, France
Corresponding Author: Conflict of interest:
Raffaele Longo, e-mail: raflongo@libero.it; r.longo@chr-metz-thionville.fr None declared

Patient: Male, 61

Final Diagnosis:

Symptoms:

Medication:

Clinical Procedure:

Specialty:

Objective:

Background:

Case Report:

Conclusions:

MeSH Keywords:

Full-text PDF:
Pituitary metastasis from renal cell carcinoma

Deterioration of visual acuity and field $\bullet$ persisting headache $\bullet$ excess thirst $\bullet$ polyuria

-

Total body CT-scan • brain MRI • trans-sphenoidal endoscopical surgery • radiotherapy • anti-angiogenic therapy

Oncology

\section{Rare disease}

Pituitary metastasis is uncommon, breast and lung cancers being the most frequent primary tumors. Renal cell carcinoma (RCC) is a rare cause of pituitary metastases, with only a few cases described to date.

We report a case of a 61-year-old man who presented with a progressive deterioration of visual acuity and field associated with a bitemporal hemianopsia. Two years ago, he underwent radical right nephrectomy for a clear cell RCC (ccRCC). The biological tests showed pan-hypopituitarism and diabetes insipidus. Brain MRI revealed a large sellar tumor lesion bilaterally infiltrating the cavernous sinuses, which was surgically resected. Histology confirmed a ccRCC pituitary metastasis. The patient received post-surgical radiotherapy. Considering the presence of concomitant extra-pituitary metastases, treatment with sunitinib was started, followed by several lines of therapy with axitinib, everolimus, and sorafenib because of tumor progression. The patient also presented with a pituitary tumor recurrence, which was treated by stereotaxic radiotherapy. He died five years after the initial diagnosis of RCC and 30 months after the diagnosis of the pituitary metastasis.

There are no standardized treatment guidelines for management of pituitary metastases. Pituitary surgery plays a role in symptom palliation, and it does not have any relevant impact on survival. Exclusive radiotherapy or stereotaxic radiotherapy could be an alternative to surgery in patients whose general condition is poor or who have concomitant extra-pituitary metastases.

Angiogenesis Inhibitors • Carcinoma, Renal Cell • Pituitary Neoplasms

http://www.amjcaserep.com/abstract/index/idArt/901032

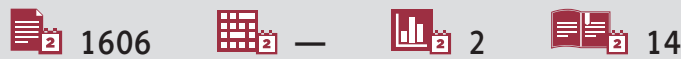




\section{Background}

Pituitary metastases are uncommon, occurring in $1 \%$ to $4 \%$ of all cancer patients in large autopsy studies, the most frequent primary tumors being breast and lung cancer [1-4]. Renal cell carcinoma (RCC) is a rare cause of pituitary metastases, with only a few cases having been reported to date [5,6]. Pituitary metastases are often asymptomatic and associated with concomitant extra-pituitary metastases [1-6]. Radiological and clinical manifestations can be characteristic and evocative but not pathognomonic [7-9]. Due to their rarity, best management of pituitary metastases is actually unknown and standardized treatment guidelines are not available [10-14].

We describe a case of a patient presenting a symptomatic pituitary metastasis from RCC.

\section{Case Report}

In June 2013, a 61-year-old Caucasian male was hospitalized for a history of progressive deterioration of visual acuity and field, associated with persistent headache, excess thirst, polyuria, and worsening of clinical conditions. In 2011, he underwent radical right nephrectomy for a clear cell RCC (CCRCC) (TNM stage I). In 2012, he received six months of neo-adjuvant hormone therapy followed by loco-regional external radiotherapy for a prostatic adenocarcinoma (TNM stage IB, Gleason score 7). Ophthalmological examination revealed bitemporal hemianopsia and reduced visual acuity (LogMAR corrected visual acuity of 0.4 and 0.3 in the right and left eyes, respectively). Endocrinological findings revealed pan-hypopituitarism associated with diabetes insipidus. Brain magnetic resonance imaging (MRI) showed a large suprasellar lesion, measuring $18 \times 34 \times 27$ $\mathrm{mm}$ in diameter, moderately heterogeneous on the pre-contrast T1- and T2-weighted images, with a hyperintense signal and necrotic characteristics in T2-weighted images and heterogeneous intense enhancement after gadolinium infusion. The lesion repressed the optic chiasm, which presented a hyperintense signal in T2-weighted MRI images and bilaterally infiltrated the cavernous sinuses (Figure 1A-1C). Total body CT scan documented 2 concomitant lung metastases and a left adrenal metastasis. In July 2013, an incomplete surgical resection of the pituitary lesion was performed by a trans-sphenoidal endoscopical approach. Histology confirmed the presence of necrotic tumor cells with clear cytoplasm and a prominent nucleolus (Figure 1D-1E). At immunohistochemistry, cancer cells were positive for CD10, cytokeratin AE1/AE3 (Figure 2A), vimentin (Figure $2 \mathrm{~B}$ ), and collagen IV, and negative for the $c y$ tokeratin 7, prolactin (Figure 2C), ACTH (Figure 2D), FSH and $\mathrm{GH}$ (Figure 2E), and TSH. This pattern was consistent with the diagnosis of a metastasis from the ccRCC. Post-surgical pituitary radiotherapy was also performed (30 Gy/10 fractions).
Replacement hormone therapy was started with levothyroxine (100 mcg/daily), hydrocortisone (30 mg/daily), and desmopressin (120 mg 3 times/daily). According to Motzer/Heng classification, the patient was classified as intermediate risk, and from October 2013 to April 2014 he received a total of 4 cycles of sunitinib. In March 2014, ophthalmological examination revealed a positive shift of the visual field and acuity. In April 2014, because of a lung tumor progression, 2nd-line treatment with axitinib was started, followed by everolimus from December 2014 to March 2015, and then by sorafenib. Because of a left eyelid ptosis with diplopia and bilateral inferior quadranopsia, a brain MRI was performed, revealing a sellar tumor recurrence spreading out to the left cavernous sinus, associated with a $16 \mathrm{~mm}$ nasal septum metastasis, which was treated in August 2015 by stereotaxic irradiation ( 5 fractions of 4 Gy). In October 2015, lung, vertebral, and brain tumor progression was observed. The pituitary recurrence and the nasal septum metastasis showed a necrotic aspect on the brain MRI. The patient received palliative brain irradiation (30 Gy/10 fractions) with a radiotherapy flash of the 11 th thoracic vertebra (8 Gy). Considering that the patient's clinical condition was quickly worsening, the best supportive care was started. The patient died of acute respiratory insufficiency in January 2016, 5 years after the initial cCRCC diagnosis and 30 months after the diagnosis of the pituitary metastasis.

\section{Discussion}

Pituitary metastasis is rare and occurred in $1 \%$ to $4 \%$ of all cancer patients in a large autopsy study [1]. It represents $0.87 \%$ of all intracranial metastases and $1.9 \%$ in the autopsy case series [1-6]. Breast and lung cancer are the most common tumors metastasizing to the hypophisis with a percentage of $37.2 \%$ and $24.2 \%$, respectively, followed by prostate (5\%) and kidney cancer (2.6\%) [1-6]. There no explanations to support this different pituitary tropism, the pituitary prolactin-rich environment probably favoring breast metastatic cells' location and growth [4].

Pituitary metastasis is usually asymptomatic. In a review of the literature concerning 31 patients, Gilard et al. observed that only $20 \%$ of patients appeared to be symptomatic [12]. As pituitary metastases often present in a disseminated cancer setting, characteristic clinical pituitary signs are usually overwhelmed by symptoms associated with concomitant extra-pituitary metastases [12].

Diabetes insipidus (45.2\%) is the most common symptom of pituitary metastases, particularly when the posterior lobe of the gland is affected. The main reason for this frequent involvement of the pituitary posterior lobe is strictly related to its wide contact with the adjacent dura mater and its direct 


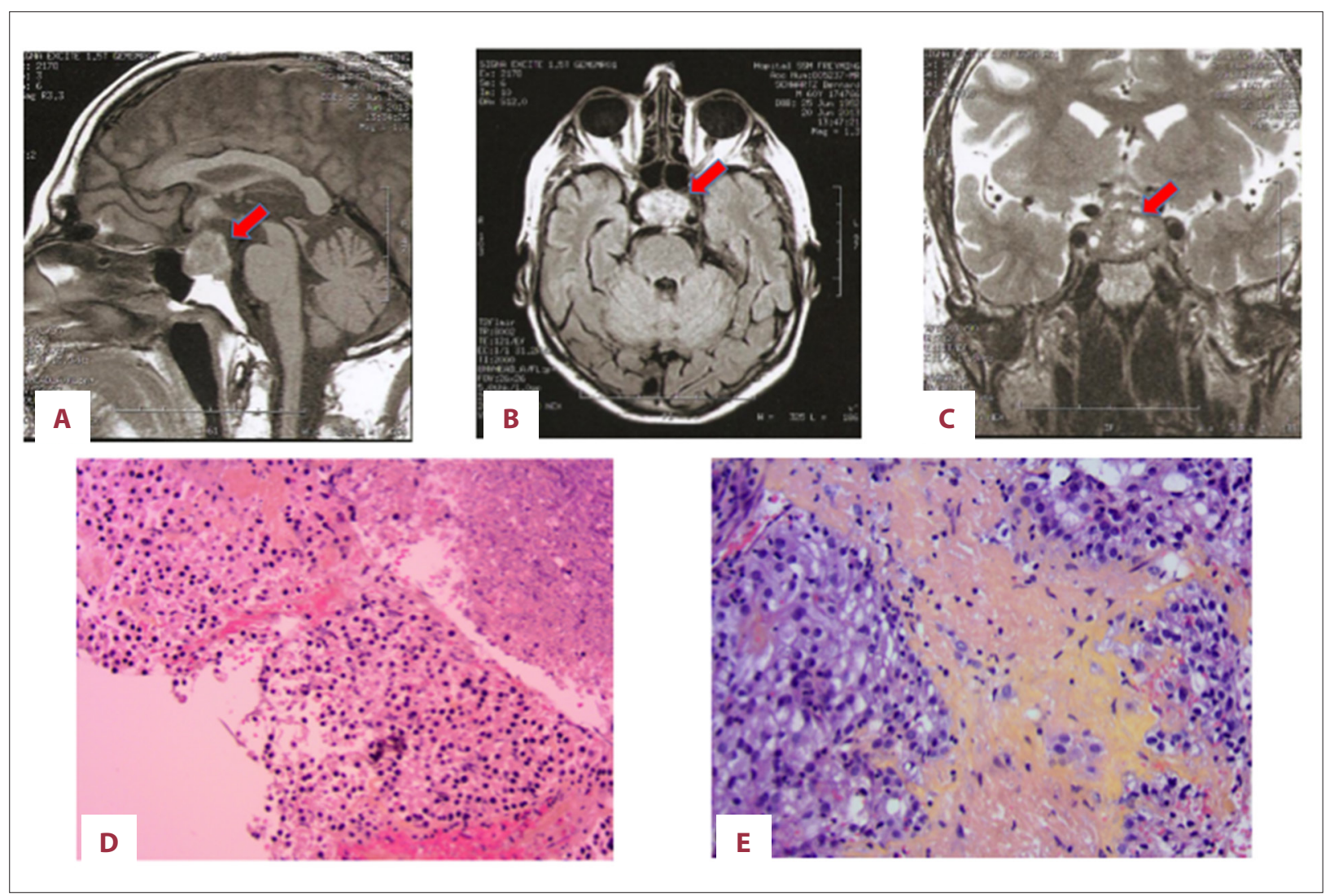

Figure 1. Brain MRI. (A) Sagittal section (T1-weighted image): heterogeneous pituitary metastasis (red arrow). (B) Axial section (FLAIR image): hyperintense pituitary lesion (red arrow). (C) Frontal section (T2-FSE image): hyperintense, cystic pituitary lesion infiltrating the optical chiasm (red arrow). (D) Histology: tumor epithelial cells with clear cytoplasm and a small, round nucleus, arranged in a compact-alveolar (nested) or acinar growth pattern separated by a delicate branching network of vascular tissue, consistent with a metastasis from a clear cell RCC (hematoxylin and eosin stain, original magnification $\times 20$ ).

(E) Hematoxylin and eosin stain, original magnification $\times 40$.

arterial supply from the hypophyseal arteries, contrasting with the anterior lobe, which is supplied from the hypophyseal portal system [7]. Diabetes insipidus is followed by visual impairment. The most common type of visual defect as first clinical presentation is cranial nerve II deficit with bilateral hemianopsia (27.9\%). In $23.6 \%$ of the cases, the first diagnosis is made on biological anterior pituitary insufficiency. Some patients (21\%) develop other cranial deficits, such as $3^{\text {rd }}$, $4^{\text {th }}$, and $6^{\text {th }}$ palsy. At diagnosis, headaches are present in $15.8 \%$ of cases, fatigue in $7.9 \%$, and hyperprolactinemia in $6.3 \%$ of patients [1-8]. A recent study revealed that anterior pituitary dysfunction is more prevalent (90\%) and diabetes insipidus less prevalent $(24 \%)$ in patients with RCC pituitary metastases compared to ones associated with other primary metastatic tumors [7]. Several reports clearly identified the tendency of RCC metastases to extend into the suprasellar region toward the optic chiasm [1-6].

The rapid onset of clinical signs and the occurrence of diabetes insipidus, which is extremely rare in pituitary adenomas, more frequently lead to a diagnosis of pituitary metastasis instead of a pituitary adenoma. In the series of Gilard et al., oculomotor palsy occurred in $42 \%$ of patients with metastasis as compared to fewer than $5 \%$ of patients with pituitary adenomas [12].

MRI is not specific for pituitary metastases, but some characteristics can be seen, such as a rapidly growing sellar and parasellar mass with erosion and/or infiltration of the sellar floor or posterior clinoid process, thickening of the pituitary stalk, loss of a high-intensity signal in the posterior pituitary lobe on T1-weighted images [7], high signal intensity on T2weighted images with iso-intense or hypointense signals, and cavernous sinus invasion [7, 9].

In several reported cases, pre-contrast images showed an homogeneous ( $\mathrm{T} 1$ ) or heterogeneous (T2) iso-intense lesion and a well-enhanced sellar mass with gadolinium, suggesting a highly vascular content [7-9]. 


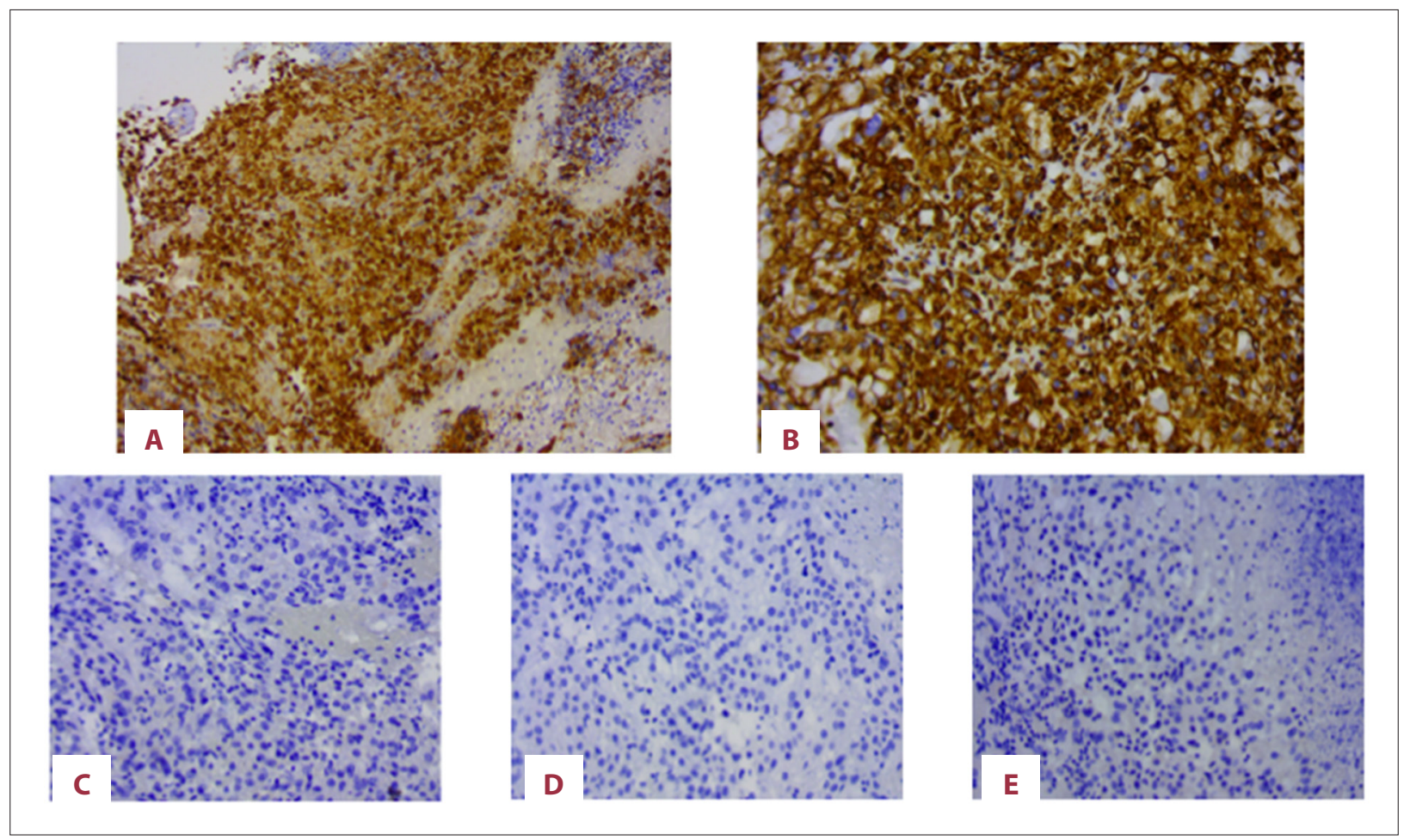

Figure 2. Immunohistochemistry. Tumor cells positive for cytokeratin AE1/AE3 (A) and vimentin (B), and negative for prolactin (C), ACTH (D), and GH (E).

18F-FDG-PET highlighted sellar tumoral hypermetabolism, associated with other systemic hypermetabolic metastasis. In a published case, 18F-FDG-PET showed no increased uptake in the sellar mass in contrast to the other metastatic lesions in the body [8]. A report revealed that various pathological abnormalities were identified using MRI in $52.7 \%$ of patients who showed focal hypophyseal 18F-FDG accumulation. The most common entity was pituitary adenoma; there was no significant difference in standardized uptake values between malignant and benign lesions [7-9].

Due to their rarity, best management of pituitary metastases is actually unknown, and there are no standardized treatment guidelines. Generally, surgery can play a role in symptom palliation and improving quality of life. Zoli et al. observed an improvement in visual deficit with surgery, without any clinically relevant modification of the endocrinology abnormalities [11]. However, surgical treatment of pituitary metastasis remains uncommon, total resection being difficult to assess because of the high tumor vascularization and local invasiveness [10-13].

In most published series, post-surgical radiotherapy was used, particularly in cases of incomplete tumor surgery [10-13]. Exclusive radiotherapy or stereotaxic radiotherapy could be an alternative to surgery in patients whose general condition is poor or who have concomitant extra-pituitary metastases [14].
Overall survival of patients with pituitary metastasis remains poor after surgery, even in cases of post-surgery radiotherapy [10-14]. Surgical removal seems to have no impact on patients' survival [10-14]. Based on the literature data, median survival is about 6-7 months after the diagnosis and is strictly correlated to tumor subtype, the patient's clinical condition, and the presence of concomitant extra-pituitary metastases. In the series of Zoli et al. concerning 15 patients suffering from pituitary metastases from any primary sites, overall survival was 11.8 months [11].

\section{Conclusions}

Pituitary metastases are uncommon and difficult to diagnose. Clinical neurological symptoms and endocrinological findings of pan-hypopituitarism associated with diabetes insipidus should suggest the presence of a pituitary metastasis in a cancer patient, particularly in a context of metastatic disease. Diagnosis needs to be confirmed by radiological imaging and histology. Treatment of pituitary metastases is not standardized and should be carefully tailored to patients' clinical conditions, tumor histology, and the presence of concomitant extra-pituitary metastases, the aims being symptom palliation and improving quality of life. 
In our case, the patient underwent incomplete surgical resection of the pituitary metastasis by a trans-sphenoid endoscopical approach, followed by sellar radiotherapy. After this treatment, improvement in the visual acuity was observed but not in the visual field. By contrast, endocrinological abnormalities and diabetes insipidus persisted after tumor excision. A pituitary progression, associated with a nasal septum metastasis, appeared 22 months after surgery. Despite several lines of systemic therapies using anti-angiogenic drugs and an mTOR inhibitor, the patient died 30 months after the diagnosis of pituitary metastasis.

\section{References:}

1. He W, Chen F, Dalm B et al: Metastatic involvement of the pituitary gland: A systematic review with pooled individual patient data analysis. Pituitary, 2015; 18: 159-68

2. Kovacs K: Metastatic cancer of the pituitary gland. Oncology, 1973; 27: 533-42

3. Komninos J, Vlassopoulou V, Protopapa D et al: Tumors metastatic to the pituitary gland: Case report and literature review. J Clin Endocrinol Metab, 2004; 89: 574-80

4. Kim YH, Lee BJ, Lee KJ, Cho JH: A case of pituitary metastasis from breast cancer that presented as left visual disturbance. J Korean Neurosurg Soc, 2012; 51: 94-97

5. Gopan T, Toms SA, Prayson RA et al: Symptomatic pituitary metastases from renal cell carcinoma. Pituitary, 2007; 10: 251-59

6. Kramer CK, Ferreira N, Silveiro SP et al: Pituitary gland metastasis from renal cell carcinoma presented as a non functioning macroadenoma. Arq Bras Endocrinol Metabol, 2010; 54: 498-501

7. Chaudhuri R, Twelves C, Cox TC, Bingham JB: MRI in diabetes insipidus due to metastatic breast carcinoma. Clin Radiol, 1992; 46: 184-88

\section{Acknowledgments}

We would like to thank Mrs. Nadia Ouamara for the English revision.

\section{Competing interest}

The authors declare no conflict of interest.

8. Hyun SH, Choi JY, Lee KH et al: Incidental focal 18F-FDG uptake in the pituitary gland: clinical significance and differential diagnostic criteria. J Nucl Med, 2011; 52: 547-50

9. Hwang JM, Kim YH, Kim TM, Park SH: Differential diagnosis and management of a pituitary mass with renal cell carcinoma. J Korean Neurosurg Soc, 2013; 54: 132-35

10. Morita A, Meyer FB, Laws ER Jr.: Symptomatic pituitary metastases. J Neurosurg, 1998; 89: 69-73

11. Zoli M, Mazzatenta D, Faustini-Fustini $M$ et al: pituitary metastases: Role of surgery. World Neurosurg, 2013; 79: 327-30

12. Gilard V, Alexandru C, Proust F et al: Pituitary metastasis: is there still a place for neurosurgical treatment ? J Neurooncol, 2016; 126: 219-24

13. Grossman R, Maimon S, Levite R, Ram Z: Multimodal treatment of hemorrhagic pituitary metastasis as first manifestation of renal cell carcinoma. World Neurosurg, 2013; 79: 798.E1-5

14. Yokoyama T, Yoshino A, Katayama Y et al: Metastatic pituitary tumor from renal cell carcinoma treated by fractionated stereotactic radiotherapy case report. Neurol Med Chir, 2004; 44: 47-52 